\title{
RATE ADAPTIVE RESOURCE ALLOCATION IN OFDMA USING BEES ALGORITHM
}

\author{
Archana C $\mathbf{C}^{\mathbf{1}}$, Rejith K.N ${ }^{2}$ \\ ${ }^{1} P G$ Scholar, ECE Department, MES College of Engineering, Kerala, India \\ ${ }^{2}$ Assoc. Professor, ECE Department, MES College of Engineering, Kerala, India
}

\begin{abstract}
Orthogonal Frequency Division Multiple Access (OFDMA) is the promising access technique for future networks which allows multiple users to transmit simultaneously. The problem of allocating resources (subcarriers, bit and power) among the communicating users in multiuser orthogonal frequency division multiplexing (OFDM) system is a constraint optimization problem. Bio-inspired Networking represents an emerging area to obtain optimal solutions for handling various challenges in the networking scenario. Nature is our mother and an inspiration from nature always gives the best. Bio-inspired algorithms are known for their efficiency in solving NP hard problems. This paper focusses on realizing the rate adaptive resource allocation problem in OFDM systems using bio-inspired approach. The resource allocation problem using Genetic Algorithm, algorithms based on foraging behavior in ants and flocking behavior in birds have already been modelled. The paper suggests the application of Bees Algorithm for resource allocation in OFDMA with the goal of maximizing the data rate of each user.
\end{abstract}

Keywords: OFDMA, Bees Algorithm, Resource allocation, and PSO

\section{INTRODUCTION}

Due to the fast development of digital signal processing and very large scale integrated circuits, wireless communication systems have been experiencing an explosive growth in the past decades. Next generation wireless networks are expected to handle large number of subscribers, while at the same time deal with the different service requirements of each user. Thus Orthogonal Frequency Division Multiple Access (OFDMA) forms the radio resource allocation scheme for the existing and envisioned networks to support the increasing number of users with the limited spectrum level. OFDMA allows several users to transmit simultaneously at lower data rates. The available spectrum band is divided into a number of sub-channels and each user is provided with a disjoint set of subcarriers. After the subcarrier allocation is determined, the bit and power allocation algorithm can be applied to each user on its allocated subcarriers. The user can transmit his data in the allocated subcarriers. A major challenge in OFDMA is that for a given number of users and subcarriers, how to allocate a disjoint set of subcarriers among the users. The classical approaches for the problem are complex and NP hard.

Researchers have turned their attention towards applying the nature inspired computational approach for solving the challenges in networking scenario [1]. Bio-inspired approach implies transferring knowledge from the natural systems to the engineering system. The future networks ranging from nano- scale communication to Inter-planetary Internet are facing challenges in the communication and managing aspects. The inherent property of the biological organisms to self-organize, learn and evolve has inspired the researchers to address the significant issues in computer networking. Bio-inspired Algorithms that mimics the behavior of biological organisms have been used to optimize complex problems [2] [3].

This paper studies the effect of applying Bees Algorithm (BA) for rate adaptive resource allocation in an OFDMA system. The problem of resource allocation in OFDMA can be handled in two ways: Rate Adaptive (RA) method and Margin adaptive (MA) method. In rate adaptive method the resource allocation is optimized by maximizing the data rate of each user while keeping the power consumption of each user below an acceptable level. Margin adaptive method minimizes the maximum power consumption of each user maintaining a rate constraint. The use of Bees algorithm for RA based allocation strategy is studied in a single cell scenario.

\section{RELATED WORKS}

Genetic algorithm (GA) was the first bio-inspired algorithm used to study the problem of resource allocation in OFDMA [4] [5]. But GA can give only sub-optimal results and includes iterative process. Ant Colony optimization (ACO) technique has been widely used to address the problem. In [6], ACO was applied for subcarrier and bit allocation for margin adaptive problem which provided better performance in terms of less transmit power consumption and faster convergence. ACO was compared with GA and other conventional algorithms for adaptive resource allocation in OFDMA [7]. But ACO requires more memory space for computation and a reduction in number of iterations are required. 
It is widely reported that Particle Swarm Optimization (PSO) [8] is very easy to implement to solve real world optimization problems. Gheitanchi et al. [9] has applied PSO for subcarrier allocation in OFDMA systems with significant reduction of computational complexity and increased flexibility compared to conventional techniques; whereas, PSO resource allocation techniques proposed by Chakravarthy et al. [10] has improved delay characteristics while maintaining fairness and throughput utilization. In [11] PSO was applied for sub-channel allocation in downlink OFDMA and provided better sum-capacity values. Ahmed [12] has shown that the Differential Evolution techniques is better than PSO but takes more time to converge. The Bees algorithm has not yet applied for the problem of resource allocation. The algorithm in literature is mentioned to be a fast converging algorithm and therefore can be useful for practical systems.

\section{SYSTEM MODEL AND PROBLEM FORMULATION}

Consider a single cell with a base station (BS) capable of serving $K$ users with $N$ subcarriers. In a downlink OFDMA system, each user estimate channel perfectly and these estimates are made known to the transmitter via a feedback channel. These channel estimates are used as inputs to the resource allocation algorithm that is allowed to run at the transmitter. The allocation module assigns subcarriers to each user according to some QoS criteria like rate or BER request. Serial data from all the users are fed into the resource allocation block at the transmitter, which then allocates bits from different users to different sub-channels. Thus the transmitter applies resource allocation algorithm to assign different subcarriers to different users and then determines the bit distribution of the user on each subcarrier assigned to it. The subcarrier allocation is made known to all users through separate control channels. The user's bits are then modulated into $N$, M-level QAM symbols which are combined using IFFT into an OFDMA symbol. This is then transmitted through a Rayleigh channel with bandwidth $B$ [13].

Let $R_{k}$ be the data rate of each user $k=1,2, \ldots \ldots . K$. Then the resource allocation strategy is based on maximizing $R_{k}$ keeping the power consumption of each user below an allowable budget. $P_{k}$ is the transmit power of each user. The channel gain of user $k$ in subcarrier $n$ is denoted by $h_{k_{2} n}$.

Then the objective function for the problem can be formulated as [14] [15],

$$
\begin{aligned}
& \text { maximize } R_{k}=\sum_{n=1}^{N} c_{k, n} r_{k, n} \\
& \text { subject to } c_{k, n} \in\{0,1\} \forall k, n \\
& \sum_{n=1}^{N} c_{k, n} p_{k, n} \leq P_{k}
\end{aligned}
$$

Where $c_{k_{2} n}$ is the subcarrier allocation indicator such that $c_{k, n}=1$ if subcarrier $n$ is assigned to user $k$ and 0 otherwise. The number of bits of user $k$ in subcarrier $n, r_{k_{n} n}$ is given by,

$$
r_{k, n}=0.5 \log _{2}\left(1+p_{k, n} H_{k, n}\right)
$$

$H_{k, n}={ }_{k, n}^{2} / \sigma_{n}{ }^{2}$ is the sub-channel SNR and $\sigma_{n}{ }^{2}$ is the noise power spectral density of AWGN channel.

\section{BEES ALGORITHM}

The Bees Algorithm is a new population-based search algorithm, first developed by Pham DT in 2005 [16]. The algorithm is inspired from the food foraging behavior of honey bees in nature. In its basic version [17] [20], the algorithm performs a kind of neighborhood search combined with random search. The processing and collection of food is highly organized in the honey bee colony. The bees can move about randomly in search of food. In nature, the food sources with large amount of nectar should be visited by more bees.

The bee colony exhibits the property of division of labor. Each group of bees is given separate designations and they work accordingly. Generally, the bee colony contains a single fertile female known as the Queen, a few thousands of males called Drones and a several thousands of sterile females called Workers. There are two types of worker bees namely- scout bees and forager bees. Worker bees are responsible for the collection of food. The foraging process begins when the scout bees move about randomly in search of promising flower patches, which constitutes an exploration phase. The bees collect information about the food patch in the exploration phase. By performing the waggle dance, scout bees share the information about the direction and distance to patches of flower and the amount of nectar within this flower with their hive mates. The bees then evaluate the food patches based on the nectar quality and energy usage. Thus more foragers will be recruited to follow that scout bee which carries information that is rated above a certain quality threshold. Forager bees move on to exploit the promising flower patches [16] [17].

In the basic Bees Algorithm (BA), one bee is one particle [16] [18]. A fixed number of bees are created and allocated randomly for each food source. The fitness at each of these points is calculated. Bees that have the highest finesses are chosen as selected bees and sites visited by them are chosen for neighborhood search. The population of bees around each food patch is updated in such a way that more number of bees move towards the food patch with maximum fitness. The steps are repeated for a fixed number of iterations. In each iteration the quality of flower exploited by more bees can deteriorate leading to a change in the allocation pattern of bees. 


\section{PROPOSED SOLUTION}

Inspired from the Bees Algorithm, the problem of resource allocation in OFDMA was handled by considering each bee as a subcarrier that has to be allocated to a user, who corresponds to the food patch. The algorithm starts by initializing a population of subcarriers for each user and then for each user, for the allocated subcarriers the fitness is evaluated [18] [19].

The flowchart of BA for resource allocation problem is shown in figure below.

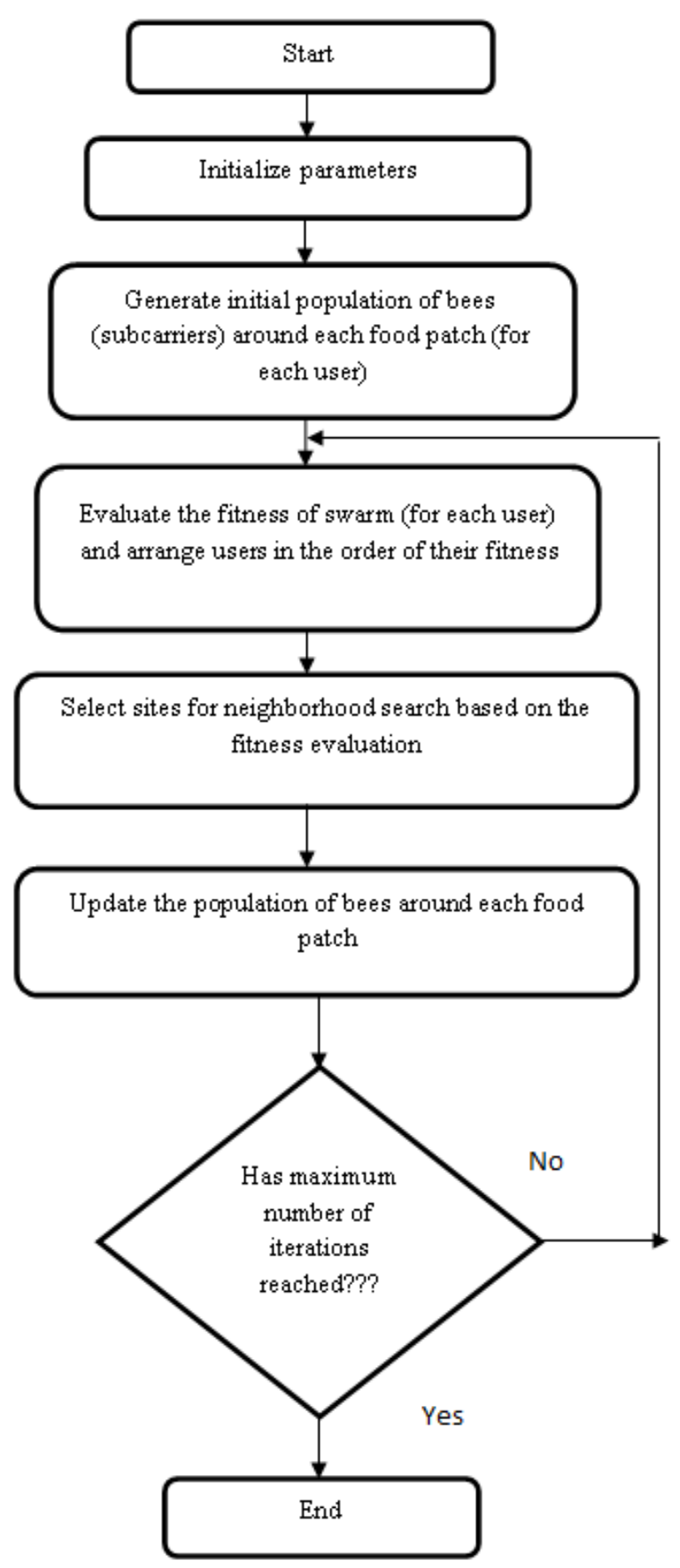

Fig-1 Flowchart of BA for OFDMA resource allocation.
The steps in the algorithm are as follows:

1. Initialize the parameters including number of users $K$, number of sub-channels $N$, and maximum number of iterations. Here $N=1024$, and $K$ was varied ranging from 8 to 16 ,

2. Initialize a population of sub-channels (bees) around each user (food patch),

3. Evaluate the fitness for each user for the subcarriers allotted (as per Eq-1),

4. Sort the list of users in the descending order of their fitness,

5. While maximum number of iterations not reached, select a user for neighborhood search,

6. Update the population of bees for the users based on neighborhood search step,

7. Evaluate the fitness with new population,

8. Run the algorithm until termination criteria is met.

Selecting the sites for neighborhood search [19] is a crucial step in the resource allocation problem. The aim of OFDMA technique is to allow several users to transmit simultaneously at lower data rates and lower transmission powers. Therefore the algorithm has to select the user with lowest data rate in the neighborhood search step. This may be slightly contradictory to what is happening in nature, but is essential to provide fairness among the users in terms of power consumption and data rate.

\section{SIMULATION RESULTS}

The use of Bees Algorithm for rate adaptive resource allocation in OFDMA was simulated in MATLAB R2013a. The number of subcarriers considered was fixed at 1024 and the number of users was varied from 8 to 18 . Total power was assumed to be $1 \mathrm{~W}$. The data rate of each user after the maximum number of iterations is shown in Fig-2 and Fig-3 for 8 and 16 users respectively.

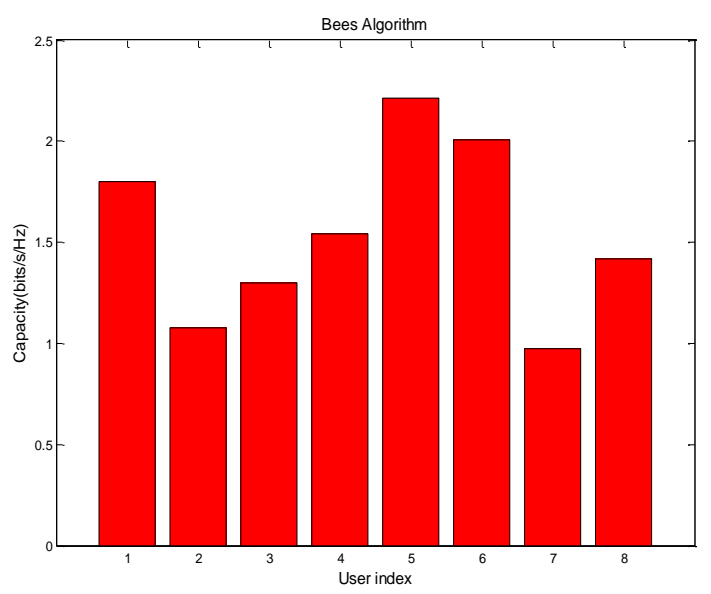

Fig-2 Capacity versus User index for 8 users.

Every user is allowed to transmit even at lower data rates. Thus the algorithm is able to support multiuser diversity, as each user see the channel differently. 


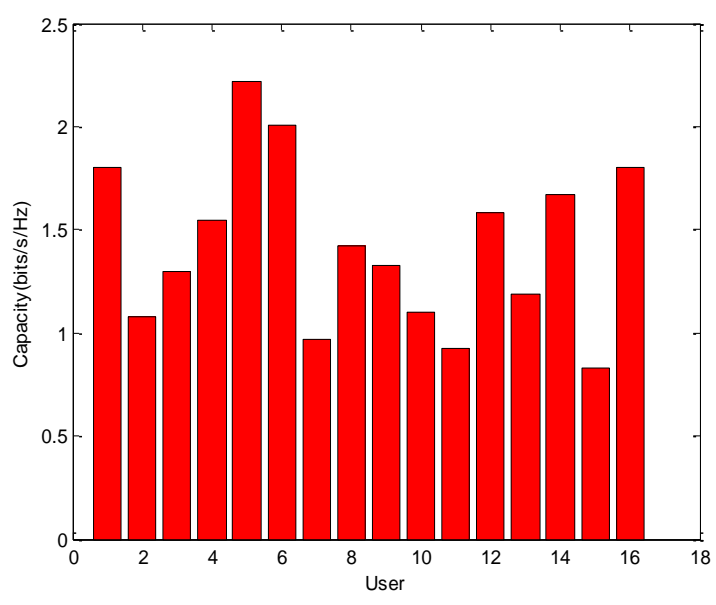

Fig-3 Capacity versus User index for 16 users.

The working of the algorithm for resource allocation problem in OFDMA can be validated by observing the variation in the average data rate for increasing number of users.

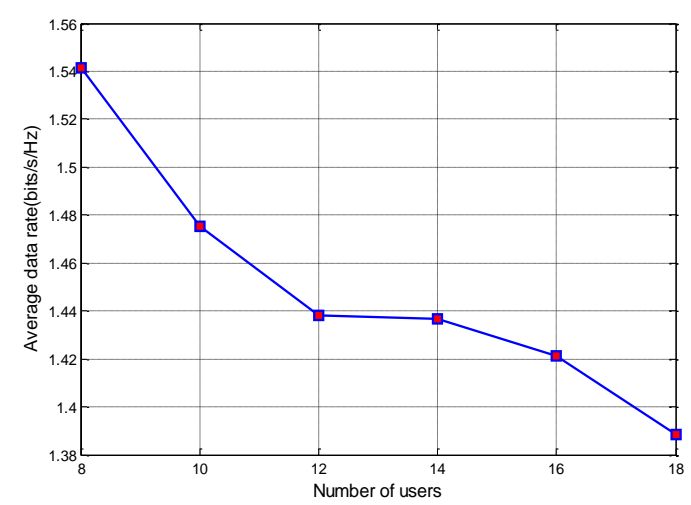

Fig-4 Variation of Average data rate with Number of users

Fig-5 shows the variation of sum-capacity with the number of users. The obtained result has been compared with the sum-capacity value obtained by applying PSO on the problem.

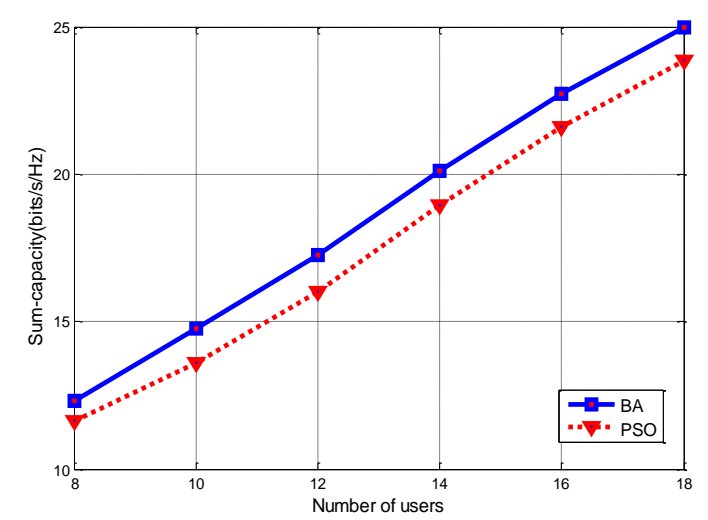

Fig-5 Comparison of BA \& PSO using Sum capacity versus Number of users plot.
It is clear that the use of BA for sub-channel allocation for OFDMA systems has consistently higher sum capacity than the method using PSO.

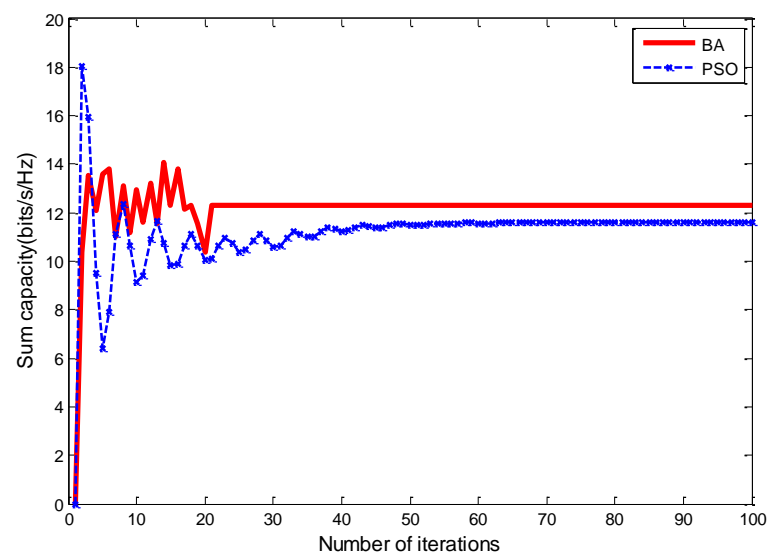

Fig-6 Variation of Sum capacity with Number of iterations for BA and PSO

In order to analyze the convergence of the algorithm, sum capacity versus number of iterations was plotted. It is evident from Fig-6 that the BA converges faster than PSO when applied for the rate adaptive resource allocation problem. BA converges at around 20 iterations while PSO around 40 iterations.

\section{CONCLUSIONS}

In this paper, the use of Bees algorithm as a new method to address the problem of rate adaptive resource allocation in OFDMA is proposed. The proposed method is easy to implement and the algorithm shows faster convergence in the rate maximization problem. The simulation results indicate that this approach is a viable alternative since it has performance comparable to PSO in terms of achieving higher total capacities. However the results obtained using the presented algorithm demands fairness in the data rate value of users.

\section{REFERENCES}

[1]. Zhongshan Zhang, Keping Long, Jianping Wang, and Falko Dressler, "On Swarm Intelligence Inspired SelfOrganized Networking: its bionic mechanisms, designing principles and optimization approaches", IEEE Communications Surveys Tutorials, vol.16, no.1, pp.513537, July 2013.

[2]. Falko Dressler and Ozgur B. Akan. "Bio-inspired Networking: From Theory to Practice" IEEE Communications Magazine, vol. 48, no. 11, pp.176-183, Nov.2010.

[3]. Falko Dressler, Ozgur B. Akan, "A survey on bioinspired networking", Elsevier Computer Networks, vol. 54, no. 6, pp.881-900, April 2010.

[4]. Nitin Sharma, Dr. K R Anupama, "A novel Genetic Algorithm for Adaptive Resource Allocation in Multiuser OFDM Systems with Proportional Rate Constraint", 
International Journal of Recent Trends in Engineering, vol 2, no. 5, Nov. 2009.

[5]. Y.B Reddy, V.Poha, "Genetic Algorithm Approach for Resource Allocation in Multi-User OFDM Systems", International Conference on Communication Systems Software and Middleware, pp. 1-6, Jan.2007.

[6]. Jungsup Song and Dong Hoi Kim, "Subcarrier and Bit Allocation Scheme for the MA Problem based on the Ant Colony Optimization to Minimize Power Consumption in OFDMA Systems", International Journal of Innovative Computing, Information and Control, vol. 7,no.8,pp. 47554764, Aug.2011.

[7]. ZHAO Ying-hong, XU Xiao-dong, HAO Zhi-jie, TAO Xiao-feng, "Adaptive subcarrier and bit allocation based on ant colony optimization", The Journal of China Universities of Posts and Telecommunications, ELSEVIER Publications, vol.17,no.6, pp. 59-64, Dec. 2010.

[8]. Eberhart R.C and Yuhui Shi, "Particle swarm optimization: developments, applications and resources", IEEE Proceedings on Evolutionary Computation, vol.1, pp.81-86, May.2001.

[9]. S.Gheitanchi et al.," Particle Swarm Optimization for Resource Allocation in OFDMA", Proc. International Conference on digital Signal Processing, 2007.

[10]. Chilukuri Kalyana Chakravarthy, Prasad Reddy, "Particle Swarm Optimization Based Approach for Resource Allocation and Scheduling in OFDMA Systems", Int. J. Communications, Network and System Sciences, vol.3, no.5, pp.466-471, 2010.

[11]. Nitin Sharma, Anand Kamat Tarcar, Varghese Antony Thomas, K.R. Anupama, "On the use of particle swarm optimization for adaptive resource allocation in orthogonal frequency division multiple access systems with proportional rate constraints", Information Sciences, vol. 182, no.1, pp.115-124, Jan. 2012.

[12]. I.Ahmed et al., "Margin Adaptive resource Allocation for Multi-user OFDM Systems by Particle Swarm Optimization and Differential Evolution", International Journal of engineering \& Technology, February 2011.

[13]. Rajendrasingh Annauth and Harry C.S.Rughooputh,"OFDM Systems Resource Allocation using Multi- Objective Particle Swarm Optimization", International Journal of Computer Networks Communications (IJCNC), vol.4, no.4, July 2012.

[14]. Vasileios D. Papoutsis and Stavros A. Kotsopoulos, "Efficient Rate Adaptive Resource Allocation Scheme in Uplink OFDMA Wireless Systems", International Conference on Wireless and Mobile Communications, 2011. [15]. Ian C.Wang, Zukang Shen, Brian L.Evans and Jeffry G.Andrews, "A Low Complexity Algorithm for Proportional Reaource Allocation in OFDMA Systems", IEEE Workshop on Signal Processing Systems,pp.1-6,2004.

[16]. D. T. Pham, E. Kog, A. Ghanbarzadeh, S. Otri, S. Rahim and M. Zaidi, "The Bees Algorithm: A Novel Tool for Complex Optimization Problems", In Proceedings of the Intelligent Production Machines and Systems (IPROMS) Conference, Pages 454461, 2006.

[17]. Fahimeh Aghazadeh and Mohammad Reza Meybodi, "Learning Bees Algorithm For optimization", International
Conference on Information and Intelligent Computing, vol.18, 2011.

[18]. Sunil Nakrani, “On Honey Bees and Dynamic Server Allocation in Internet Hosting Centers", Adaptive Behavior Journal, vol.2, no.4, pp. 223-240, 2004.

[19]. Muhammad Rashid, "Combining PSO Algorithm and Honey Bee Food Foraging Behavior for Solving Multimodal and Dynamic Optimization Problems", National University of Computer Emerging Sciences, Pakistan.Feb.2010.

[20]. Baris Yuce, Michael S. Packianather, Ernesto Mastrocinque, Duc Truong Pham 4and Alfredo Lambiase, "Honey Bees Inspired Optimization Method: The Bees Algorithm”, Insects, vol.4, no.4, pp.646-662, 2013.

\section{BIOGRAPHIES}

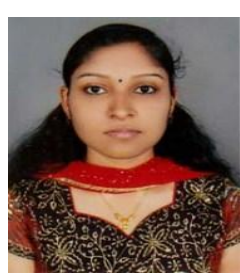

The author received the Bachelor degree in Electronics and Communication Engineering from Calicut University in 2011. She is currently a PG scholar in Communication Engineering and Signal Processing at ECE Department of MES College of Engineering, Kuttipuram. Her Project area is Bio-inspired Networking.

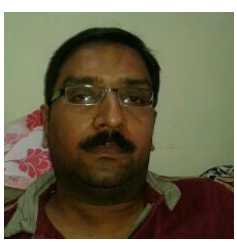

The author is currently working as Associate Professor at ECE Department in MES College of Engineering. He received Bachelor degree in Electronics and Communication from LBS College of Engineering, Kasaragod in 1998 and Master degree in Digital Electronics and Advanced Communication from MIT Manipal in 2008. He is currently doing $\mathrm{PhD}$ under Karpagam University. His area of research is Bio-inspired Networking. 